\title{
Simulating Crowd Evacuation in a Social Force Model with Iterative Extended State Observer
}

\author{
Juan Wei $\mathbb{D}^{1,2}$ Wenjie Fan, ${ }^{2}$ Zhongyu Li, ${ }^{1,3}$ Yangyong Guo $\mathbb{D}^{1,3}$ Yuanyuan Fang, \\ and Jierui Wang ${ }^{1}$ \\ ${ }^{1}$ College of Computer Science, Chengdu Normal University, Chengdu 611130, China \\ ${ }^{2}$ Key Laboratory of Pattern Recognition and Intelligent Information Processing, \\ Institutions of Higher Education of Sichuan Province, Chengdu University, Chengdu 610106, China \\ ${ }^{3}$ Key Laboratory of Interior Layout Optimization and Security Institutions of Higher Education of Sichuan Province, \\ Chengdu Normal University, Chengdu 611130, China \\ Correspondence should be addressed to Yangyong Guo; jonathan923@protonmail.com
}

Received 23 September 2019; Accepted 19 May 2020; Published 1 August 2020

Academic Editor: Jose E. Naranjo

Copyright (c) 2020 Juan Wei et al. This is an open access article distributed under the Creative Commons Attribution License, which permits unrestricted use, distribution, and reproduction in any medium, provided the original work is properly cited.

\begin{abstract}
Due to the interaction and external interference, the crowds will constantly and dynamically adjust their evacuation path in the evacuation process to achieve the purpose of rapid evacuation. The information from previous process can be used to modify the current evacuation control information to achieve a better evacuation effect, and iterative learning control can achieve an effective prediction of the expected path within a limited running time. In order to depict this process, the social force model is improved based on an iterative extended state observer so that the crowds can move along the optimal evacuation path. First, the objective function of the optimal evacuation path is established in the improved model, and an iterative extended state observer is designed to get the estimated value. Second, the above model is verified through simulation experiments. The results show that, as the number of iterations increases, the evacuation time shows a trend of first decreasing and then increasing.
\end{abstract}

\section{Introduction}

Crowd evacuation is a complex system. Due to the limitations of evacuation time, space, and obstacles, as well as the interaction between crowds and spontaneous behavior of the individual, there are some phenomena such as competition for safe exit space and mutual pushing in the evacuation process. Once the crowd state changes abruptly, it is extremely easy to lead to secondary disasters, e.g., crowding and trampling. Therefore, the analysis of crowd evacuation process and the study of evacuation behavior are the key links to guarantee the evacuation safety and improve the ability of congestion prevention and control.

Researchers have proposed a variety of crowd motion models from different perspectives, which can be divided into macromodel and micromodel. Macromodel mainly studies the macrocharacteristics, e.g., crowd flow, but neglects the motion state of individuals, and its representative model is the fluid mechanics model $[1,2]$. Micromodel focuses on the modeling of an individual behavior, and its representative models are cellular automaton, lattice gas, and social force models [3-12]. As a common continuous micromodel, the social force model is proposed on the basis of the concept of social force combined with classical Newtonian mechanics and has some advantages that discrete models such as cellular automaton do not have, e.g., arbitrary turning of crowds and different degrees of overlapping generated between crowds, which makes the crowds simulated by the social force model closer to reality. However, there are still some limitations in the social force model; for example, when the pedestrian density is too high or too low, some unrealistic phenomena often occur. In this regard, researchers have proposed a large number of improvement methods based on the social force model. Helbing and Molnár [13] discussed the corresponding force concept in more detail and had applied to the description of other behaviors, and computer simulations of crowds of interacting crowds show that the social force model is capable of 
describing the self-organization of several observed collective effects of crowd behavior very realistically. Gerta et al. [14] suggested a very simple mollified version of the social force model that conserves the desired dynamic properties of the original many-body system but elegantly and cost-efficiently resolves several of the issues concerning stability and numerical resolution. Liu et al. [15] proposed a video datadriven social force model for simulating crowd evacuation, in which fitness function was defined to model the behavior of a pedestrian goal selection, and a new force called "group force" was added to the primary social force model. Kretz [16] investigated under which circumstances, parameter choices, and social force model variants oscillations did occur and how this could be prevented. It is shown that oscillations can be excluded if the model parameters fulfill certain relations. Liu [17] developed a social force model to study the crowd evacuation when a terrorist attack occurs in the public place, and the simulation results showed that the emergency exit choice strategy has an advantage over the ordinary exit choice strategy in daily life for reducing casualties. The more unbalanced the terrorists' initial distribution around the exits is, the more noticeable this advantage will be. Rinke et al. [18] developed a multilayer approach for representing the movement of road users and their interaction based on the social force model. In a freeflow layer, a realistic path is calculated for each user towards his destination, while a conflict layer is used for detecting possible conflict situations and computing an appropriate reaction. Xu et al. [19] proposed a novel technique termed miSFM for the simulation of crowd evacuation with mutual information and the social force model, and the key innovation lies in highlighting how the dynamic adjustment of SFM parameters reveals much more realistic crowd movements for the evacuation simulation. Anvari et al. [20] presented a three-layered microscopic mathematical model, which was capable of representing the behavior of pedestrians and vehicles in shared space layouts. In the second layer, the social force model is modified and extended for mixed traffic to produce feasible trajectories. Zhang et al. [21] proposed a modified two-layer social force model to simulate and reproduce a group gathering process based on low-density group organization patterns, and the experiment result also showed that a stable group pattern and a suitable leader could decrease collision and allow a safer evacuation process.

Although such problems as arbitrary turning and overlapping of crowds have been solved, the social force model can effectively describe the "self-organization" and "arching" phenomena. However, there are still some shortcomings, especially the pedestrian's exclusion behavior is not completely consistent with the pedestrian's actual motion, and crowds are completely under the control of forces, and pedestrian's intelligent behavior is rarely considered, which oversimplifies the pedestrian's path discovery process. Iterative learning is suitable for objects with repetitive motion properties, and the previous control error is used to modify the current control amount, thereby improving the prediction accuracy. In this paper, the optimal evacuation path is used as an unknown expectation, and the expected control is calculated by repeated iterations. The social force model is improved in this paper using an iterative extended state observer by defining the objective function of the optimal evacuation path.

\section{Social Force Model}

In order to effectively describe the random fluctuation effect generated by crowds in the evacuation process, the random fluctuation force is introduced in this paper on the basis of the traditional social force model, and the kinetic equation of a single pedestrian is described as follows:

$$
m_{i} \frac{\mathrm{d} V_{i}}{\mathrm{~d} \tau}=\sum_{q} f_{i q}+\sum_{j \neq i} f_{i j}+\sum_{w} f_{i w}
$$

where $f_{i q}, f_{i j}$, and $f_{i w}$ are driving force, repulsive force, and attraction, respectively, $v_{i}$ represents the expected velocity, and $m_{i}$ represents the quality of the pedestrian $i . \tau$ is a time dimension variable introduced to transform the existing velocity vector difference into acceleration. The smaller the $\tau$ is, the greater the acceleration caused by crowds will be.

If the initial pedestrian velocity is $v_{i}^{0}$ and the relaxation time used to correct the motion velocity of pedestrian is $\tau_{i}$, then the driving force $f_{i q}$ can be calculated as per the following equation:

$$
\sum_{q} f_{i q}=m_{i} \frac{v_{i}^{0}(t) e_{i}^{0}(t)-v_{i}(t)}{\tau_{i}} .
$$

If there are multiple exits, the pedestrian's initial pedestrian velocity is calculated according to the direction between the current position and the each exit. The minimum value is used as the final initial pedestrian velocity of the pedestrian.

At the same time, the repulsive force $f_{i j}$ between the pedestrian $\alpha$ and the pedestrian $\beta$ mainly includes psychosocial force $\vec{f}_{\alpha \beta}^{s o c}(t)$ and physical contact force $\vec{f}_{\alpha \beta}^{p h}(t)$, which can be calculated as follows:

$$
\sum_{j \neq i} f_{i j}=\vec{f}_{\alpha \beta}^{s o c}(t)+\vec{f}_{\alpha \beta}^{p h}(t) .
$$

In addition, the position vector difference between the pedestrian $a$ and attraction point $i$ is $\vec{r}_{a i}=\vec{r}_{a}-\vec{r}_{i}$, in which $W_{a_{i}}\left(\left\|\vec{r}_{a_{i}}\right\|, t\right)$ represents a monotonic increasing potential function similar to the repulsion effect; then, the attraction $f_{i w}$ can be calculated as follows:

$$
\sum_{w} f_{i w}=-\nabla W_{a_{i}}\left(\left\|\vec{r}_{a_{i}}\right\|, t\right) .
$$

\section{The Improved SFM}

Due to the effect of external environment and personal factors, crowds will constantly adjust their evacuation path in the evacuation process to achieve the purpose of rapid evacuation. In order to depict this process, the social force model is improved based on an iterative extended state observer in this study, named I-SFM (iterative extended state 


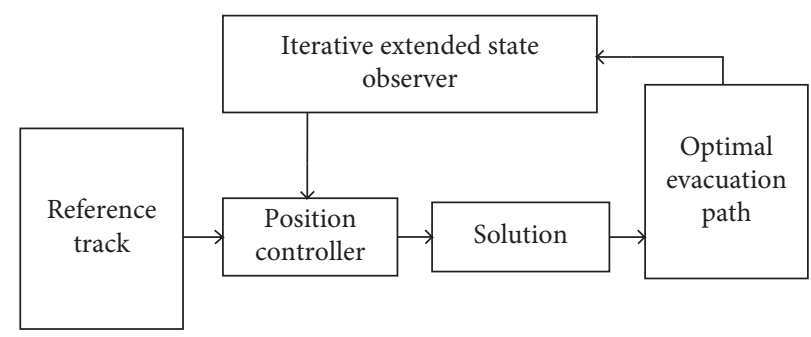

FIgUre 1: Calculation method.

observer-social force model). The system can continuously learn from it, so that the pedestrian evacuation path can proceed along the optimal iteration direction. Moreover, the control parameters in the improved model only contain the partial derivatives of the nonlinear system with respect to path parameters, so its dynamic complexity is reduced and can be estimated more easily, thus improving the simulation performance of the model.

For a repetitive system of evacuation simulation, the information from previous process can be used to modify the current evacuation control information to achieve a better evacuation effect, and iterative learning control can achieve an effective prediction of the expected path within a limited running time. The calculation method of an expanded state observer is shown in Figure 1.

Here, the effect on crowds at the moment $t+1$ is as follows:

$$
\begin{aligned}
f\left(y_{k}(t+1)\right)= & f\left(y_{k}(t), y_{k}(t-1), \ldots, y_{k}\left(t-n_{y}\right), u_{k}(t),\right. \\
& \left.u_{k}(t-1), \ldots, u_{k}\left(t-n_{u}\right)\right)+d_{k}(t+1),
\end{aligned}
$$

where $u_{k}(t)$ and $y_{k}(t)$, respectively, represent the optimal evacuation path and the actual evacuation path of crowds, $k$ represents the number of iterations, and $f(\cdot)$ represents the social force encountered during evacuation; $n_{y}$ and $n_{u}$ represent unknown time steps; $d_{k}(t+1)$ represents the other disturbance factors on pedestrians, which can be considered as the total of other disturbance factors not considered in this paper. When the number of iterations tends to be infinite, the trajectory error $e_{k}(t)=y_{d}(t)-y_{k}(t)$ converges to zero in the whole finite time interval, that is, $\lim _{k \rightarrow \infty} e_{k}(t)=0$.

The following objective function of the optimal evacuation path is taken into account:

$$
Z\left(u_{k}(t)\right)=\left|e_{k}(t+1)\right|^{2}+\lambda\left|u_{k}(t)-u_{k-1}(t)\right|^{2},
$$

where $\lambda(\lambda>0)$ represents the weight factor. Considering the evacuation system running in finite time, when the partial derivative of the optimal evacuation path $u_{k}(t)$ is continuous and bounded, there must be a partial derivative $\Phi_{k}(t)$ and a nonlinear term $\xi_{k}(t)$, which can be converted into the following form equivalently:

$$
\Delta u_{k}(t+1)=\phi_{k}(t) \Delta u_{k}(t)+\xi_{k}(t) .
$$

By substituting equation (6) into equation (7), in which $\mu(\mu \in(0,1])$ represents the step length factor, we solve the partial derivative of the optimal evacuation path $u_{k}(t)$ and make it equal to 0 to get

$$
u_{k}(t)=u_{k-1}(t)+\frac{\mu \phi_{k}(t)\left(e_{k-1}(t+1)-\xi_{k}(t)\right)}{\lambda+\phi_{k}^{2}(t)} .
$$

$\Phi_{k}(t)$ is unknown in the optimal learning control rate, so it needs to be estimated. The estimated value $\hat{\phi}_{k}(t)$ is as follows:

$$
\begin{aligned}
\hat{\phi}_{k}(t)= & \hat{\phi}_{k-1}(t)+\eta \Delta u_{k-1}(t) \\
& \frac{\left(\Delta y_{k-1}(t+1)-\hat{\phi}_{k-1}(t) \Delta u_{k-1}(t)-\xi_{k-1}(t)\right)}{\lambda+\Delta u_{k-1}^{2}(t)}
\end{aligned}
$$

where $\eta(0<\eta<2)$ represents the weight factor.

$\xi_{k}(t)$ is unknown in the above formula, so it needs to be estimated. The gain of adjustable observer is $\alpha$, and the limiting constant is $b_{\xi}$. In this paper, an iterative extended state observer is designed to obtain its estimated value $\hat{\xi}_{k}(t)$ :

$$
\left\{\begin{array}{l}
\hat{\xi}_{0, k}(t)=\hat{\xi}_{k-1}(t)-\alpha\left(y_{d}(t+1)-y_{k-1}(t+1)\right) \\
\hat{\xi}_{k}(t)=\left\{\begin{array}{l}
b_{\xi}, \hat{\xi}_{0, k(t)}>b_{\xi} \\
\hat{\xi}_{0, k(t)},\left|\hat{\xi}_{0, k(t)}\right| \leq b_{\xi} \\
-b_{\xi}, \hat{\xi}_{0, k(t)}<b_{\xi}
\end{array}\right.
\end{array}\right.
$$

Thus, the optimal evacuation path can be further obtained:

$$
u_{k}(t)=u_{k-1}(t)+\frac{\mu \hat{\phi}_{k}(t)\left(e_{k-1}(t+1)-\hat{\xi}_{k}(t)\right)}{\lambda+\hat{\phi}_{k-1}^{2}(t)},
$$

where 


$$
\begin{aligned}
& \int \hat{\phi}_{k}(t)=\hat{\phi}_{0}(t), \quad \text { if } \quad \hat{\phi}_{k}(t) \leq \varepsilon \quad \text { or } \quad\left|\Delta u_{k-1}(t)\right| \leq \varepsilon, \\
& \left\{\hat{\phi}_{k}(t)=\hat{\phi}_{k-1}(t)+\eta \Delta u_{k-1}(t) \frac{\Delta y_{k-1}(t+1)-\hat{\phi}_{k-1}(t) \Delta u_{k-1}(t)-\hat{\xi}_{k-1}(t)}{\lambda+\Delta u_{k-1}^{2}(t)}, \quad\right. \text { other } \\
& \left\{\begin{array}{l}
\hat{\xi}_{0, k}(t)=\hat{\xi}_{k-1}(t)-\alpha\left(y_{d}(t+1)-y_{k-1}(t+1)\right) \\
\hat{\xi}_{k}(t)=\left\{\begin{array}{l}
b_{\xi}, \hat{\xi}_{0, k(t)}>b_{\xi}, \\
\hat{\xi}_{0, k(t)},\left|\hat{\xi}_{0, k(t)}\right| \leq b_{\xi}, \\
-b_{\xi}, \hat{\xi}_{0, k(t)}<-b_{\xi},
\end{array}\right.
\end{array}\right.
\end{aligned}
$$

where $\hat{\phi}_{0}(t)$ represents the initial value of $\hat{\phi}_{k}(t)$, and $\varepsilon$ represents any small normal number.

Thus, the social force model based on an iterative extended state observer can be established:

$$
m_{i} \frac{\mathrm{d} V_{i}}{\mathrm{~d} \tau}=\sum_{q} f_{i q}+\sum_{j \neq i} f_{i j}+\sum_{w} f_{i w}+f\left(u_{k}(t)\right) .
$$

\section{Mathematical Simulations}

According to the above improved social force model, a simulation platform is established for verification. The simulation environment parameters are set here: the evacuation space is $50 \mathrm{~m} * 50 \mathrm{~m}$, the initial crowd density is $\rho=0.4$ (which is defined as the ratio of the current number of pedestrians to the area of evacuation space), the expected velocity is $\nu=1.45 \mathrm{~m} / \mathrm{s}$, and $\lambda=5, \mu=0.6, \eta=1$. At the initial evacuation stage, crowds are randomly and evenly distributed in the evacuation space and begin to evacuate to the exit after the simulation begins. First, the improved social force model I-SFM is used for experimental analysis in this paper, and the simulation process is shown in Figure 2.

Meanwhile, the traditional social force model (traditional SFM) and the modification of the self-stopping social force model (self-stopping SFM) [12] are applied to the experimental platform, and its calculation results are compared with I-SFM. The calculation results of the above two models are compared with the mean values of ten simulation processes. Figure 3 shows the relationship between the number of evacuees and the evacuation time of traditional social force model SFM, self-stopping SFM, and our improved social force model I-SFM. As can be seen from Figure 3, there is little difference between the number of evacuees in three models at the initial evacuation stage. However, when the evacuation time exceeds $10 \mathrm{~s}$, the number of evacuees in SFM is obviously low, while selfstopping SFM and I-SFM can complete the evacuation in a relatively short time. This is because I-SFM can constantly adjust the evacuation path for crowds to avoid excessive congestion when the simulation time is $10 \mathrm{~s}$ or so, and there is a congestion nearby the exit due to a large number of crowds. At the same time, Figure 4 shows the relationship between the sum of pedestrian's moving path length and the evacuation time of models SFM and I-SFM. As can be seen from Figure 4, the sum of pedestrian's moving path length in I-SFM is much larger than that in SFM, which also indicates that our algorithm can continuously adjust the pedestrian evacuation path and direction in the evacuation process, and thus increase the moving path length.

Meanwhile, the output results of the model are observed by changing the number of iterations in the improved model to test the robustness of the improved model I-SFM in a better way. Here, 100 iterations are taken as the standard evacuation time $T_{\gamma}$, and the error of other iterative evacuation time $T_{i}$ is defined as error $=\left|T_{i}-T_{\gamma}\right| * 100 / T_{\gamma}$. Figure 5 shows the change between the evacuation time error and the number of evacuees under different iterations. As can be seen from Figure 5, under the same number of evacuees, the more the number of iterations is, the smaller the error will be. But this does not mean that the error can be reduced by blindly increasing the number of iterations. Table 1 shows the variation trend of the average error of more iterations when the number of evacuees is 100 . When the number of iterations reaches $110-130$, the average error is reduced to a minimum. And too many or too few iterations corresponding to the average error are greater than the value in this range. This means that a moderate number of iterations is conducive to improving the effectiveness of the model. However, when the number of evacuees reaches 110 , there is a sudden change in each iteration. This means that in this evacuation space, there are many better evacuation route choices by 110 evacuees, which has a greater impact on model accuracy.

Finally, the number of evacuees is 100. Figure 6 shows the change between the evacuation time and the number of iterations at different expected velocities. As can be seen from Figure 6, as the number of iterations increases, the evacuation time shows a trend of first decreasing and then increasing, which is consistent with the conclusion in 


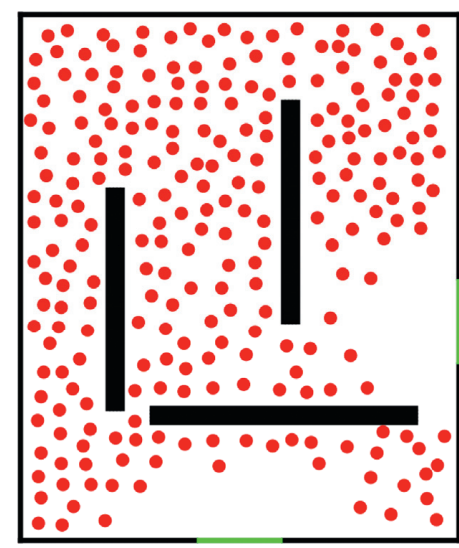

(a)

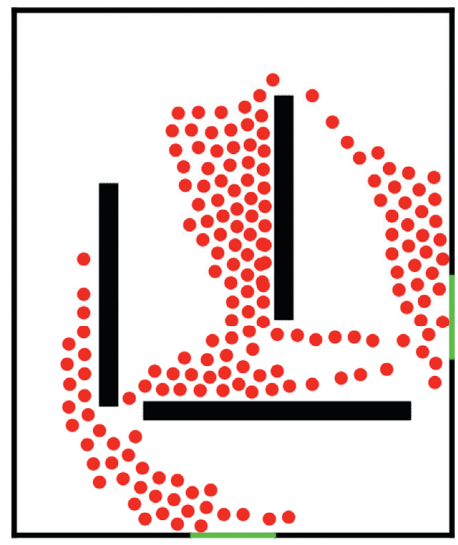

(d)

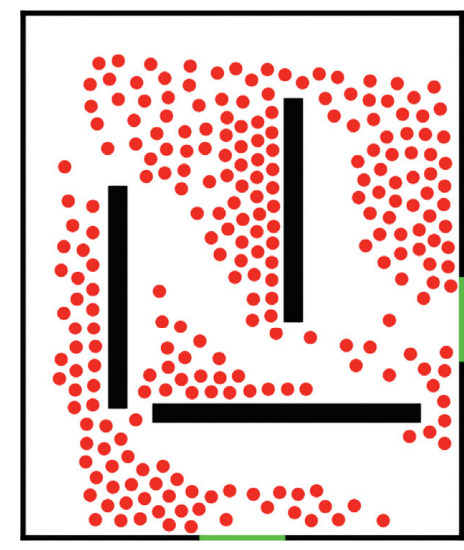

(b)

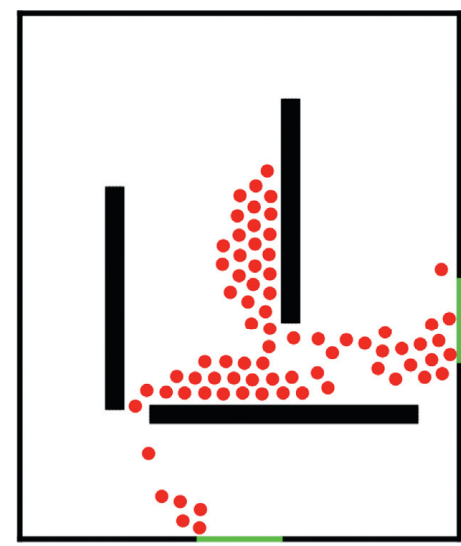

(e)

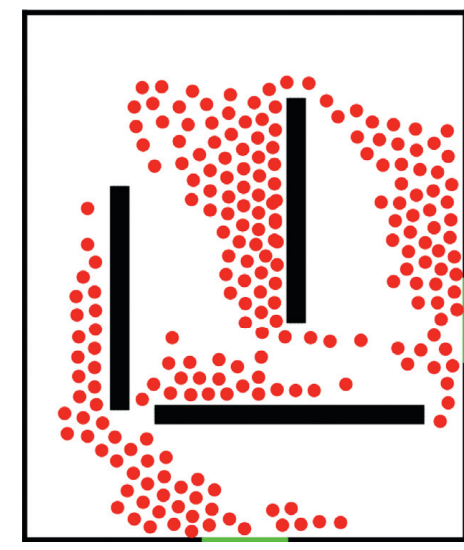

(c)

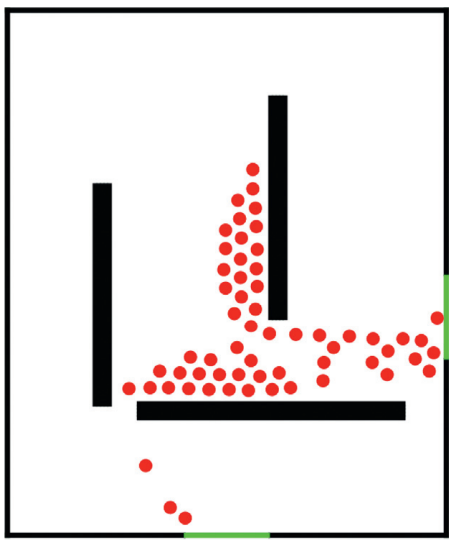

(f)

Figure 2: Simulation process. (a) $t=1 \mathrm{~s}$. (b) $t=7 \mathrm{~s}$. (c) $t=11 \mathrm{~s}$. (d) $t=15 \mathrm{~s}$. (e) $t=19 \mathrm{~s}$. (f) $t=22 \mathrm{~s}$.

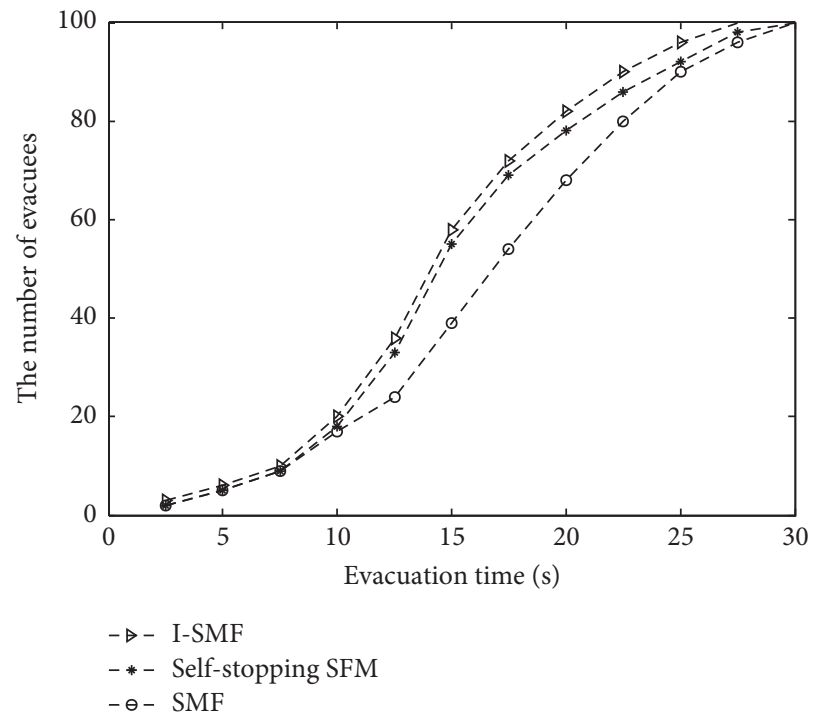

FIgURE 3: Contrastive analysis of the number of evacuees and evacuation time. 


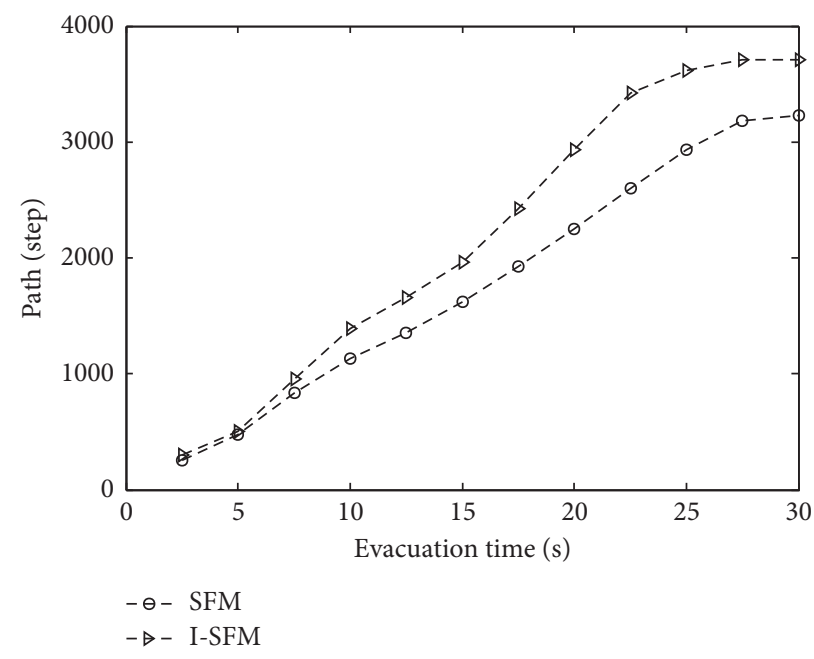

FIGURE 4: The relationship between the sum of pedestrian's moving path length and the evacuation time.

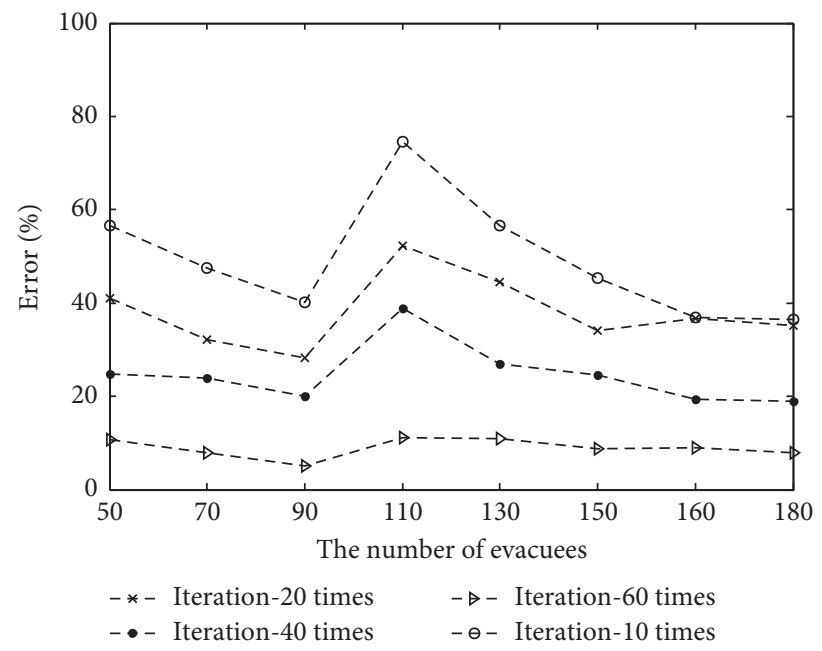

FIgURE 5: The change between the evacuation time error and the number of evacuees under different iterations.

TABLE 1: The trend of the average error in different iterations.

\begin{tabular}{lc}
\hline The number of iterations & Average error $(\%)$ \\
\hline 70 & 36 \\
80 & 32 \\
90 & 27 \\
110 & 24 \\
120 & 23 \\
130 & 24 \\
140 & 26 \\
150 & 27 \\
160 & 29 \\
170 & 30 \\
180 & 33 \\
\hline
\end{tabular}

Figure 5; that is, the number of iterations is reduced to a minimum near 110. In addition, we can observe that, under the same number of iterations, it is not that the higher the expected velocity is, the shorter the evacuation time will be. For example, the evacuation time with expected

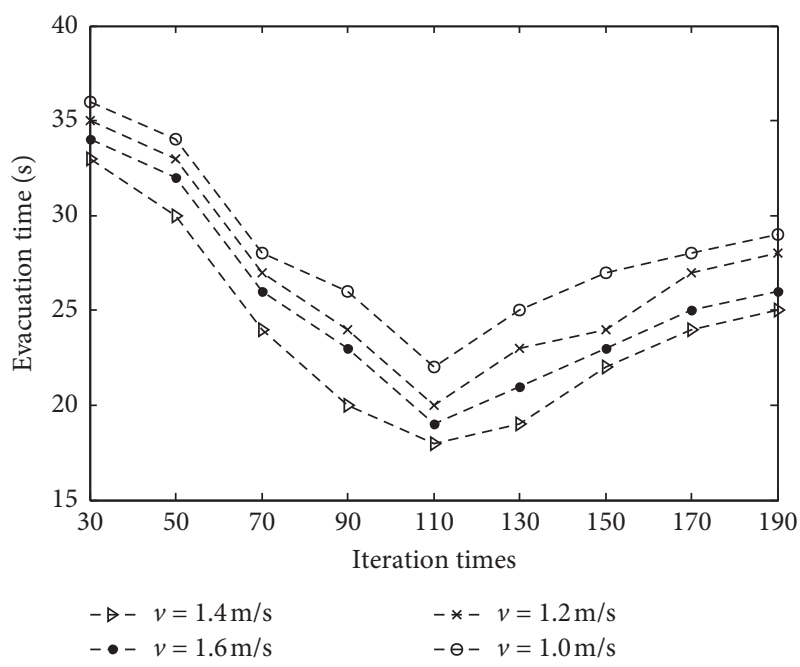

FIgURE 6: The change between the evacuation time and the number of iterations at different expected velocities. 
velocity $v=1.4 \mathrm{~m} / \mathrm{s}$ is less than that of expected velocity $v=1.6 \mathrm{~m} / \mathrm{s}$, which coincides with the phenomenon of "faster is slower."

\section{Conclusions}

Since the description of pedestrian's exclusion behavior and actual motion is not completely consistent in the traditional social force model, crowds are completely under the control of forces, and little consideration is given to pedestrian's intelligent behavior, the pedestrian's path discovery process is oversimplified. In order to effectively depict the phenomenon that crowds adjust their evacuation path dynamically in the evacuation process, the social force model is improved based on an iterative extended state observer in this paper, so that the crowds can move along the optimal evacuation path. This model first gives the objective function of the optimal evacuation path, and an iterative extended state observer is designed to get the estimated value. At the same time, the above model is verified through simulation experiments. The results show that (1) in the effective iterations, the more the number of iterations is for the same number of evacuees, the smaller the error will be, and (2) as the number of iterations increases, the evacuation time shows a trend of first decreasing and then increasing. Under the same number of iterations, it is not expected that the higher the expected velocity is, the shorter the evacuation time will be.

\section{Data Availability}

The data used to support the findings of this study are included within the article.

\section{Conflicts of Interest}

The authors declare that they have no conflicts of interest.

\section{Acknowledgments}

This research was supported by the Scientific Research Foundation of Science and Technology Department of Sichuan Province of China (Grant no. 2018JY0129), the Scientific Research Fund of Sichuan Provincial Education Department of China (Grant no. 15TD0038), and the Scientific Research Fund of Key Laboratory of Pattern Recognition and Intelligent Information Processing of Chengdu University (Grant no. MSSB-2019-06).

\section{References}

[1] C. Estelle and L. Ludovic, "A macroscopic theory for unsignalized intersections," Transportation Research Part B: Methodological, vol. 41, no. 10, pp. 1139-1150, 2007.

[2] L. F. Henderson, "On the fluid mechanics of human crowd motion," Transportation Research, vol. 8, no. 6, pp. 509-515, 1974.

[3] D. Helbing, I. Farkas, and T. Vicsek, "Simulating dynamical features of escape panic," Nature, vol. 407, no. 6803, pp. 487-490, 2000.
[4] W. Zeng, P. Chen, H. Nakamura, and M. Iryo-Asano, “Application of social force model to pedestrian behavior analysis at signalized crosswalk," Transportation Research Part C: Emerging Technologies, vol. 40, pp. 143-159, 2014.

[5] L. Li, H. Liu, and Y. Haun, "Arch formation-based congestion alleviation for crowd evacuation," Transportation Research Part C: Emerging Technologies, vol. 100, pp. 88-106, 2019.

[6] A. Poulos, F. Tocornal, J. C. de la Llera, and J. Mitrani-Reiser, "Validation of an agent-based building evacuation model with a school drill," Transportation Research Part C: Emerging Technologies, vol. 97, pp. 82-95, 2018.

[7] P. Mitrani-Reiser and M. Bukáček, "Influence of agents heterogeneity in cellular model of evacuation," Journal of Computational Science, vol. 21, pp. 486-493, 2017.

[8] J. Wei, H. Zhang, Y. Guo, and M. Gu, "Experiment of bidirection pedestrian flow with three-dimensional cellular automata," Physics Letters A, vol. 379, no. 16-17, pp. 1081-1086, 2015.

[9] C. Burstedde, K. Klauck, A. Schadschneide, and J. Zittartz, "Simulation of pedestrian dynamics using a two-dimensional cellular automaton," Physica A: Statistical Mechanics and Its Applications, vol. 295, no. 3-4, pp. 507-525, 2001.

[10] J. Hu, L. You, H. Zhang, J. Wei, and Y. Guo, "Study on queueing behavior in pedestrian evacuation by extended cellular automata model," Physica A: Statistical Mechanics and Its Applications, vol. 489, no. 1, pp. 112-127, 2018.

[11] R. Alizadeh, "A dynamic cellular automaton model for evacuation process with obstacles," Safety Science, vol. 49, no. 2, pp. 315-323, 2011.

[12] D. R. Parisi, M. Gilman, and H. Moldovan, "A modification of the social force model can reproduce experimental data of pedestrian flows in normal conditions," Physica A: Statistical Mechanics and Its Applications, vol. 388, no. 17, pp. 36003608, 2009.

[13] D. Helbing and P. Molnár, "Social force model for pedestrian dynamics," Physical Review E, vol. 51, no. 5, pp. 4282-4286, 1995.

[14] G. Köster, F. Treml, and M. Gödel, "Avoiding numerical pitfalls in social force models," Physical Review E, vol. 87, p. 63305, 2013.

[15] B. Liu, H. Liu, H. Zhang, and X. Qin, "A social force evacuation model driven by video data," Simulation Modelling Practice and Theory, vol. 84, pp. 190-203, 2018.

[16] T. Kretz, "On oscillations in the social force model," Physica A: Statistical Mechanics and Its Applications, vol. 438, pp. 272-285, 2015.

[17] Q. Liu, "A social force model for the crowd evacuation in a terrorist attack," Physica A: Statistical Mechanics and Its Applications, vol. 502, pp. 315-330, 2018.

[18] N. Rinke, C. Schiermeyer, F. Pascucci, V. Berkhahn, and B. Friedrich, "A multi-layer social force approach to model interactions in shared spaces using collision prediction," Transportation Research Procedia, vol. 25, pp. 1249-1267, 2017.

[19] M. Xu, Y. Wu, P. Lv, H. Jiang, M. Luo, and Y. Ye, "miSFM: on combination of mutual information and social force model towards simulating crowd evacuation," Neurocomputing, vol. 168, pp. 529-537, 2015.

[20] B. Anvari, M. G. H. Bell, A. Sivakumar, and W. Y. Ochieng, "Modelling shared space users via rule-based social force model," Transportation Research Part C: Emerging Technologies, vol. 51, pp. 83-103, 2015.

[21] H. Zhang, H. Liu, X. Qin, and B. Liu, "Modified two-layer social force model for emergency earthquake evacuation," Physica A: Statistical Mechanics and Its Applications, vol. 492, pp. 1107-1119, 2018. 